\title{
ON SOME PROJECTION METHODS FOR APPROXIMATING FIXED POINTS OF NONLINEAR EQUATIONS IN BANACH SPACE
}

\author{
IOANNIS K. ARGYROS
}

\begin{abstract}
We use a Newton-like method to approximate a fixed point of a nonlinear operator equation in a Banach space. Our iterates are computed at each step by solving a linear algebraic system of finite order.
\end{abstract}

\section{Introduction.}

Consider the problem of approximating a fixed point $x^{\star}$ of the operator equation

$$
x=T(x)
$$

where $T(x)$ is a nonlinear operator defined on a subset $D$ of a Banach space $E$ with values in a Banach space $\hat{E}$.

We study the convergence of the Newton-like methods

$$
x_{n+1}=T\left(x_{n}\right)-P T^{\prime}\left(x_{n}\right)\left(x_{n}-x_{n+1}\right), \quad n \geq 0
$$

and

$$
y_{n+1}=T\left(y_{n}\right)-P T^{\prime}\left(x_{0}\right)\left(y_{n}-y_{n+1}\right), \quad x_{0}=y_{0}, \quad n \geq 0
$$

to $x^{\star}$, where $T^{\prime}\left(x_{n}\right)$ is the Fréchet derivative of $T$ evaluated at $x_{n}$ and $P$ is a linear projection operator projecting $E$ on its subspace $E_{p}$. If $E_{p}$ is a finite-dimensional space with $\operatorname{dim}\left(E_{p}\right)=N$, then the iterates (2) and (3) can be computed at each step by solving a system of linear algebraic equations of order at most $N$. The case when $P=I$, the identity operator on $E$, has been examined by many authors, under different assumptions $[1],[3],[4],[5],[7]$. The iterates, however, can rarely be computed in infinite dimensional spaces, since it may be very difficult or impossible to find the inverses of the linear operators $I-T^{\prime}\left(x_{n}\right), n \geq 0$. The case when $T$ is a continuous linear operator has been examined in [5], [6]. We assume that $T$ is a nonlinear operator. Our conditions are easier to verify than the ones in [5], even in the linear case.

Received Oct. 24, 1989.

Key words and phrases: Fixed point, Banach space, Neton-like method.

1980 AMS classification codes: 47D15, 47H17, 65. 
In this paper, we provide sufficient conditions for the convergence of iterations (2) and (3) to a locally unique fixed point $x^{\star}$ of equation (1).

Finally, we illustrate our results with an example.

II. Convergence theorems.

We can now formulate our main theorem concerning iteration (2).

Theorem 1. Let $T: D \subset E \rightarrow \hat{E}$ and assume

(a) the inverse of the linear operator $I-P T^{\prime}\left(x_{0}\right)$ exists and

$$
\left\|\left(I-P T^{\prime}\left(x_{0}\right)\right)^{-1}\left(x_{0}-T\left(x_{0}\right)\right)\right\| \leq \eta ;
$$

(b) the following inequalities are true for all

$$
\begin{gathered}
x, y \in U\left(x_{0}, r\right)=\left\{x \in E \mid\left\|x-x_{0}\right\|<r\right\}: \\
\left\|\left(I-P T^{\prime}\left(x_{0}\right)\right)^{-1}\left(P T^{\prime}(x)-P T^{\prime}(y)\right)\right\| \leq M\|x-y\|^{\lambda}
\end{gathered}
$$

and

$$
\left\|\left(I-P T^{\prime}\left(x_{0}\right)\right)^{-1}(Q T(x)-Q T(y))\right\| \leq q\|x-y\|^{\lambda}, Q=I-P, \lambda \in[0,1) .
$$

(c) The conditions

$$
\begin{gathered}
(\eta d)^{\lambda}<1, \\
M r^{\lambda}<1, \\
\eta+\frac{e d^{-1}}{1-e} \leq r
\end{gathered}
$$

are satisfied, where

$$
e=(d \eta)^{\lambda}, d^{\lambda-1}=c
$$

and

$$
c(r)=c=\frac{1}{1-M r^{\lambda}}\left(\frac{2 M r}{1+\lambda}+q\right) .
$$

(d) The ball $\bar{U}\left(x_{0}, r\right) \subset D$. Then, equation (1) has a fixed point $x^{*}$ in $\bar{U}\left(x_{0}, r\right)$ where $r$ is chosen to be the minimum number $r>0$ satisfying (8)-(9). Moreover, the following estimates are true

$$
\left\|x_{n}-x^{\star}\right\| \leq d^{-1} \frac{e^{n}}{1-e}, n \geq 0
$$

and

$$
\left\|x_{n+1}-x_{n}\right\| \leq c\left\|x_{n}-x_{n-1}\right\|^{\lambda}, n \geq 1
$$


Furthermore, if

$$
(d r)^{\lambda}<1
$$

then $x^{*}$ is the unique fixed point of equation (1) in $\bar{U}\left(x_{0}, r\right)$.

Proof. From (2) and (3) we get the identity

$$
\left(I-P T^{\prime}\left(x_{n}\right)\right)\left(x_{n+1}-x_{n}\right)=T\left(x_{n}\right)-T\left(x_{n-1}\right)-P T^{\prime}\left(x_{n-1}\right)\left(x_{n}-x_{n-1}\right), n \geq 1 .
$$

By the Banach lemma on invertible operators, (5) and (8), it follows that $I-P T^{\prime}(x)$ is invertible for all $x \in U\left(x_{0}, r\right)$ and

$$
\left\|\left(I-P T^{\prime}(x)\right)^{-1}\left(I-P T^{\prime}\left(x_{0}\right)\right)\right\| \leq \frac{1}{1-M\left\|x-x_{0}\right\|^{\lambda}} \leq \frac{1}{1-M r^{\lambda}} .
$$

Let us assume that $x_{0}, x_{1}, \ldots, x_{n} \in U\left(x_{0}, r\right)$, then from (4)-(6), (13) and (14) we get

$$
\left\|x_{n+1}-x_{n}\right\|
$$

$\leq\left\|\left(I-P T^{\prime}\left(x_{n}\right)\right)^{-1}\left(I-P T^{\prime}\left(x_{0}\right)\right)\right\|\left[\|\left(I-P T^{\prime}\left(x_{0}\right)\right)^{-1}\left(P T\left(x_{n}\right)-P T\left(x_{n-1}\right)\right.\right.$

$$
\left.\left.-P T^{\prime}\left(x_{n-1}\right)\left(x_{n}-x_{n-1}\right)\right)\|+\|\left(I-P T^{\prime}\left(x_{0}\right)\right)^{-1}\left(Q T\left(x_{n}\right)-Q T\left(x_{n-1}\right)\right) \|\right]
$$

$\leq \frac{1}{1-M r^{\lambda}}\left[\|\left(I-P T^{\prime}\left(x_{0}\right)\right)^{-1} \int_{0}^{1} P T^{\prime}\left(x_{n-1}+t\left(x_{n}-x_{n-1}\right)\right)-P T^{\prime}\left(x_{n-1}\right)\right)\left(x_{n}-x_{n-1}\right) d t \|$ $\left.+q\left\|x_{n}-x_{n-1}\right\|^{\lambda}\right]$

$\leq \frac{1}{1-M r^{\lambda}}\left[\frac{M}{1+\lambda}\left\|x_{n}-x_{n-1}\right\|+q\right]\left\|x_{n}-x_{n-1}\right\|^{\lambda}$

$\leq c\left\|x_{n}-x_{n-1}\right\|^{\lambda}$, which shows (11).

From (11), we get

$$
\begin{aligned}
\left\|x_{0}-x_{n+1}\right\| & \leq\left\|x_{1}-x_{0}\right\|+\left\|x_{2}-x_{1}\right\|+\cdots+\left\|x_{n}-x_{n+1}\right\| \\
& \leq \eta+c \eta^{\lambda}+c^{1+\lambda} \eta^{\lambda^{2}}+\cdots+c^{1+\lambda+\cdots+\lambda^{n-1}} \eta^{\lambda^{n}} \\
& \leq \eta+d^{-1}\left[(d \eta)^{\lambda}+(d \eta)^{\lambda^{2}}+\cdots+(d \eta)^{\lambda^{n}}\right] \\
& \leq \eta+d^{-1}\left[(d \eta)^{\lambda}+(d \eta)^{2 \lambda}+\cdots+(d \eta)^{n \lambda}\right] \\
& \leq \eta+d^{-1} e\left(1+e+e^{2}+\cdots+e^{n-1}\right) \\
& \leq \eta+d^{-1} e \frac{1-e^{n}}{1-e} \leq \eta+d^{-1} e \frac{1}{1-e} \leq r
\end{aligned}
$$

Hence, $x_{n+1} \in U\left(x_{0}, r\right)$. For $p \geq 1$,

$$
\begin{aligned}
\left\|x_{n}-x_{n+1 p}\right\| & \leq\left\|x_{n}-x_{n+1}\right\|+\left\|x_{n+1}-x_{n+2}\right\|+\cdots+\left\|x_{n+p-1}-x_{n+p}\right\| \\
& \leq d^{-1}(d \eta)^{\lambda^{n}}+d^{-1}(d \eta)^{\lambda^{n+1}}+\cdots+d^{-1}(d \eta)^{\lambda^{n+p}} \\
& \leq d^{-1} e^{n}\left[1+e+\cdots+e^{p-1}\right]=d^{-1} e^{n} \frac{1-e^{p}}{1-e} .
\end{aligned}
$$


It now follows from that the sequence $\left\{x_{n}\right\}$ is a Cauchy sequence in a Banach space and as such it converges to some $x^{\star} \in \bar{U}\left(x_{0}, r\right)$. By letting $p \rightarrow \infty$ in (16) we obtain (10), whereas by letting $n \rightarrow \infty$ in (2) we get $x^{\star}=T\left(x^{\star}\right)$. To show uniqueness let us assume that $z^{\star}$ is any fixed point of $T$ in $U\left(x_{0}, r\right)$ and use the identity

$$
\left(I-P T^{\prime}\left(x_{n}\right)\right)\left(x_{n+1}-z^{\star}\right)=T\left(x_{n}\right)-T\left(z^{\star}\right)-P T^{\prime}\left(x_{n}\right)\left(x_{n}-z^{\star}\right)
$$

to get

$$
\left\|x_{n+1}-z^{\star}\right\| \leq c\left\|x_{n}-z^{\star}\right\|^{\lambda} \leq \cdots \leq d^{-1}(d r)^{\lambda^{n}} \leq d^{-1}(d r)^{\lambda^{n}} \rightarrow 0
$$

as $n \rightarrow \infty$ from (12). Hence $x^{\star}=\lim _{n \rightarrow \infty} x_{n}=z^{\star}$.

That completes the proof of the theorem.

Note that for $\lambda=1$ the proof of the previous theorem can be repeated, but (7) becomes $c<1$, (9) becomes $\frac{\eta}{1-c} \leq r, e=c,(10)$ becomes $\left\|x_{n}-x^{\star}\right\| \leq \frac{e^{n}}{1-e} \eta$ and (12)
becomes $c<1$.

the proof of the following theorem concerning iteration (3) is omitted as similar to the proof of theorem 1 .

Theorem 2. Let $T: D \subset E \rightarrow \hat{E}$ and assume

(a) the following inequalities are true:

$$
\begin{gathered}
\left\|\left(I-P T^{\prime}\left(x_{0}\right)\right)^{-1}\left(x_{0}-T\left(x_{0}\right)\right)\right\| \leq \eta \\
\|\left(I-P T^{\prime}\left(x_{0}\right)\right)^{-1}\left(P T^{\prime}(x)-P T^{\prime}(y)\|\leq M\| x-y \|^{\lambda}\right.
\end{gathered}
$$

and

$$
\begin{gathered}
\|\left(I-P T^{\prime}\left(x_{0}\right)\right)^{-1}\left(Q T(x)-Q T(y)\|\leq q\| x-y \|^{\lambda},\right. \\
Q=I-P, \lambda \in[0,1), \text { for all } x, y \in U\left(x_{0}, R\right) .
\end{gathered}
$$

(b) The conditions

$$
\begin{gathered}
\left(\eta d_{1}\right)^{\lambda}<1, \\
\eta+\frac{e_{1} d_{1}^{-1}}{1-e_{1}} \leq R
\end{gathered}
$$

are satisfied, where

$$
e_{1}=\left(d_{1} \eta\right)^{\lambda}, d_{1}^{\lambda-1}=c_{1}
$$

and

$$
c_{1}(r)=c_{1}=2^{1-\lambda} M R+q .
$$

(c) The ball $\bar{U}\left(x_{0}, R\right) \subset D$. 
Then equation (1) has a fixed point $x^{\star}$ in $\bar{U}\left(x_{0}, R\right)$ where $R$ is chosen to be the minimum number $R>0$ satisfying conditions (b). Moreover, the following estimates are true

$$
\left\|y_{n}-x^{\star}\right\| \leq d_{1}^{-1} \frac{e_{1}^{n}}{1-e_{1}}, n \geq 0
$$

and

$$
\left\|y_{n+1}-y_{n}\right\| \leq c_{1}\left\|y_{n}-y_{n-1}\right\|^{\lambda}, n \geq 1
$$

Furthermore if

$$
\left(d_{1} R\right)^{\lambda} \leq 1
$$

then $x^{\star}$ is the unique fixed point of equation $(1)$ in $\bar{U}\left(x_{0}, R\right)$.

Note that a remark similar to the one made after Theorem 1 for the case $\lambda=1 \mathrm{can}$ now easily follow for Theorem 2 .

We now complete this paper with an application.

\section{Applications.}

Let us consider the following system in $E=\hat{E}=R^{k}$,

$$
v_{i}=f_{i}\left(v_{1}, \ldots, v_{k}\right), i=1,2, \cdots, k \text {. }
$$

Set

$$
\begin{aligned}
& T(v)\left\{f_{i}\left(v_{1}, \ldots, v_{k}\right)\right\}, i=1,2, \cdots, k \text {; } \\
& T^{\prime}(W) v=\left\{\sum_{j=1}^{k} f_{i j}^{\prime}\left(w_{1}, \ldots, w_{k}\right) v_{j}\right\}, i=1,2, \cdots, k ; \\
& P T^{\prime}(w) v= \begin{cases}\sum_{j=1}^{k} f_{i j}^{\prime}\left(w_{1}, \cdots, w_{k}\right) v_{j}, & i=1,2, \cdots, N \\
0, & i=N+1, \cdots, k,\end{cases}
\end{aligned}
$$

where the symbol $f_{i j}^{\prime}$ denotes $\partial f_{i} / \partial v_{j}$.

Iterations (2) and (3) can be written as

$$
\begin{aligned}
& v_{i, n+1}=f_{i}\left(v_{1, n}, \ldots, v_{k, n}\right)+\sum_{j=1}^{k} f_{i j}^{\prime}\left(v_{1, n}, \ldots, v_{k, n}\right)\left(v_{j, n+1}-v_{j, n}\right), i=1, \cdots, N(18) \\
& v_{i, n+1}=f_{i}\left(v_{1, n}, \cdots, v_{k, n}\right), i=N+1, \cdots, k
\end{aligned}
$$

and

$$
\begin{aligned}
& \bar{v}_{i, n+1}=f_{i}\left(\bar{v}_{1, n}, \ldots, \bar{v}_{k, n}\right)+\sum_{j=1}^{k} f_{i j}^{\prime}\left(\bar{v}_{1,0}, \ldots, \bar{v}_{n, 0}\right)\left(\bar{v}_{j, n+1}-\bar{v}_{j, n}\right), i=1, \cdots, N(19) \\
& \bar{v}_{i, n+1}=f_{i}\left(\bar{v}_{1, n}, \cdots, \bar{v}_{k, n}\right), i=N+1, \cdots, k
\end{aligned}
$$


respectively. we have

If the determinants $D\left(x_{n}\right)$ and $D_{0}$ of (18) and (19) respectively, are nonzero, then

for system (18) and

$$
\begin{gathered}
v_{i, n+1}=\frac{\sum_{m=1}^{N} D_{i m}\left(v_{n}\right) \bar{f}_{m}\left(v_{n}\right)}{D\left(v_{n}\right)}, i=1,2, \cdots, N \\
v_{i, n+1}=f_{i}\left(v_{n}\right), i=N+1, \cdots, k
\end{gathered}
$$

$$
\begin{gathered}
\bar{v}_{i, n+1}=\frac{\sum_{m=1}^{N} D_{i m}\left(\bar{v}_{0}\right) \bar{f}_{m}\left(v_{0}\right)}{\left.D_{0}\right)}, i=1,2, \cdots, N \\
\bar{v}_{i, n+1}=f_{i}\left(\bar{v}_{n}\right), i=N+1, \cdots, k
\end{gathered}
$$

for system (19).

Here

$$
\begin{aligned}
& \bar{f}_{m}\left(v_{n}\right)=f_{m}\left(v_{n}\right)-\sum_{i=1}^{k} f_{m j}^{\prime}\left(v_{n}\right) v_{j, n}+\sum_{i=N+1}^{k} f_{m j}^{\prime}\left(v_{n}\right) f_{j}\left(v_{n}\right), \\
& \bar{f}_{m}\left(v_{0}\right)=f_{m}\left(v_{0}\right)-\sum_{j=1}^{k} f_{m j}^{\prime}\left(v_{0}\right) v_{j, n}+\sum_{i=N+1}^{k} f_{m j}^{\prime}\left(v_{0}\right) f_{j}\left(v_{0}\right),
\end{aligned}
$$

$m=1,2, \cdots, k$, where $D_{i m}\left(v_{n}\right), D_{i m}\left(v_{0}\right)$ are the cofactors of the elements at the itersection of the $m$-th row and $i$-th column of the determinants $D\left(x_{n}\right)$ and $D_{0}$, respectively. eration.

We assume that the following conditions are satisfied on some region under consid-

$$
\begin{gathered}
\left|f_{i}\left(v_{i}, \ldots, v_{k}\right)-f_{i}\left(w_{1}, \ldots, w_{k}\right)\right| \leq \sum_{j=1}^{k} t_{i j}\left|v_{j}-w_{j}\right|^{\lambda}, i=N+1, \cdots, k, \lambda \in[0,1] \\
\left|f_{i j}^{\prime}\left(v_{i}, \ldots, v_{k}\right)-f_{i j}^{\prime}\left(w_{1}, \ldots, w_{k}\right)\right| \leq \sum_{s=1}^{k} b_{i j s}\left|v_{s}-w_{s}\right|^{\lambda}, i=1, \cdots, N, j=1, \cdots, k, \\
\left|D_{i m}(v)\right| \leq a_{i m^{\prime}}|D(v)| \leq a, \\
\left|f_{i j}^{\prime}(v)\right| \leq h_{i j}, i=1, \ldots, N, j=1,2, \cdots, k .
\end{gathered}
$$

For any $v \in E$, set $\|v\|=\sup _{1 \leq i \leq k}\left|v_{i}\right|$, then the constants $q$ and $M$ appearing in the Theorem 1-2 can be computed by

$$
q \leq \sup _{i=N+1, \cdots, k} \sum_{j=1}^{k} t_{i j} \text { and } M \leq \sup _{i=1,2, \cdots, N} \sum_{j, j=1}^{k} c_{i j s} .
$$

References 
[1] Argyros, I.K. "Newton-like methods under mild differentiablity conditions with error analysis," Bull. Austral, Math. Soc., Vol. 37, 2, (1987), 131-147.

[2] - "Concerning the approximate solutions of operator equations in Hilbert space under mild differentiability conditions", Tamkang J. Math. Vol. 19, 4, (1985), 7-19.

[3] Dennis, J.E., "Toward a unified convergence theory of Newton-like methods", In Nonlinear Functional Analysis and Applications (edited by L.B. Rall), Academic Press, New York, 1971.

[4] Kantorovich, L.V., "The method of successive approximation for functional equations", Acta Math. 71, (1939), 63-97.

[5] Kurnel, N.S. and Migovich, F.M., "Some Generalizations of the Newton-Kantorovich method", Ukrainskii Mathematicheskiz Zhurnal, Vol. 21, No. 5 (1969), 948-960.

[6] Ortega, J.M. and Rheinboldt, W.C., Iterative solution for nonlinear equations in several variables. Academic Press, New York, 1970.

[7] Zabreiko, P.P. and Nguen, D.F., "The majorant method in the theory of Newton-Kantorovich approximations and the Ptâk error esimates", Numer. Funct. Anal. and Optimiz. 9 (5 and 6), (1987), 671-684.

Department of Mathematical Sciences, New Mexico State University, Las Cruces, NM 88003. 